\title{
Anticoagulación en terapia de reemplazo renal continua
}

\author{
Lilia Rizo-Topete ${ }^{1}$ y Luis Augusto Juncos² \\ ${ }^{1}$ Profesora Adscrita al Servicio de Nefrología del Hospital Universitario Dr. José Eleuterio González, Monterrey, N.L., México; ${ }^{2}$ Profesor de Medicina, \\ de Fisiología y Biofísica, Central Arkansas Veterans Healthcare Systems y University of Arkansas for Medical Science, Little Rock, Arkansas, EE.UU.
}

\begin{abstract}
Resumen
La terapia de reemplazo renal continua (TRRC) se desarrolló para cubrir los inconvenientes de la terapia intermitente en pacientes críticos. Cuando la TRRC es verdaderamente continua, puede proveer de un control metabólico y de volumen sumamente efectivo. Sin embargo, el fallo prematuro o la coagulación del circuito extracorpóreo durante las terapias continúa siendo un obstáculo común que contribuye a tratamientos inadecuados, perdidas sanguíneas, altos costos y una gran cantidad de tiempo invertido por el personal de enfermería dedicado a instalar los equipos en lugar de la atención directa al paciente. El fallo temprano de los circuitos está relacionado a diversos factores, incluyendo aquellos relacionados a) con el paciente (p. ej., el paciente críticamente enfermo con lesión renal aguda (LRA) desarrolla un estado procoagulable), b) con las terapias utilizadas en estos pacientes ( $p$. ej., transfusiones), y c) con factores relacionados a la prescripción de TRRC. Por lo tanto, estrategias destinadas a prevenir el fallo del circuito deben tener en cuenta todos estos factores e incluir la optimización del funcionamiento del catéter, circuito y prescripción de TRRC. Mientras las optimizaciones de todos estos factores mejoran la vida media del circuito, la anticoagulación es generalmente también requerida para maximizar la vida del circuito. Estas estrategias de anticoagulación pueden administrarse por vía sistémica o regional (intracircuito). El objetivo de esta revisión es ofrecer un panorama general de las medidas de anticoagulación más utilizadas en TRRC.
\end{abstract}

PALABRAS CLAVE: Anticoagulación. Terapia de reemplazo renal. Lesión renal aguda. Citrato.

\begin{abstract}
Continuous renal replacement therapies (CRRT) were developed to overcome shortcomings of intermittent haemodialysis in critically ill patients. When CRRT is truly continuous, it provides very effective volume and metabolic control. However, premature loss of extracorporeal circuit patency during CRRT remains a common obstacle and contributes to inadequate treatment, and increased blood loss, costs in nursing time dedicated to CRRT instead of direct patient care. Early loss of circuit patency is related to numerous factors including (a) patient related factors (e.g. critically ill patients with acute kidney injury develop a pro-coagulant state), (b) therapy-related factors (e.g. blood transfusions), and (c) factors related to the CRRT prescription. Thus, strategies implemented to prolong circuit patency must take all these factors into account, particularly those related to CRRT; that is, to optimize the circuit life of the CRRT circuit, one must implement a comprehensive strategy that includes optimization of the catheter, circuit, and CRRT prescription. While optimization of these factors will improve circuit life, anticoagulation is generally also required to maximize it. This can be accomplished via systemic or regional (intra-circuit) anticoagulation strategies. This paper provides an overview of the most commonly used anticoagulation strategies being used in CRRT.
\end{abstract}

KEY WORDS: Anticoagulation. Continuous renal replacement therapy. Acute kidney injury. Citrate.

Correspondencia:

Lilia Rizo-Topete

E-mail: dra.liliarizo@gmail.com
Fecha de recepción: 21-11-2017

Fecha de aceptación: 16-02-2017

DOI: 10.24875/GMM.M18000066
Gac Med Mex. 2018;Supp 1:61-71

Disponible en PubMed www.gacetamedicademexico.com 


\section{Introducción}

El uso de la TRRC se ha expandido globalmente en las últimas dos décadas. Gracias a los grandes avances tecnológicos se ha convertido en el tratamiento de elección para la LRA en el paciente críticamente enfermo ${ }^{1}$. Sin embargo, permanece una resistencia a implementarlo en forma generalizada, por la percepción de que es una terapia de altos costo y complejidad, que precisa amplios recursos humanos y económicos ${ }^{2-4}$. Estos obstáculos se pueden minimizar implementando protocolos estandarizados, que incluyen una estrategia de anticoagulación simple y efectiva, y un programa de monitoreo de calidad, para determinar la eficacia de la estrategia ${ }^{1-6}$.

Uno de los principales desafíos de la TRRC es mantener la funcionalidad del circuito en forma continua durante al menos 24 horas $^{7-10}$. La interrupción prematura es muy frecuente y se han reportado casos de pacientes que solo reciben 16 horas de tratamiento en promedio por día o menos del $70 \%$ de la dosis prescripta (Fig. 1) ${ }^{10}$. Aunque la interrupción temprana de la TRRC generalmente se manifiesta como coagulación del circuito, los factores que contribuyen a la coagulación son diversos (Tabla 1) 7,9. Por lo tanto, para mejorar la vida media de un circuito debemos asegurarnos de implementar un protocolo de prescripción de TRRC que incorpore medidas que consideren todos los factores que contribuyen al mantenimiento de la funcionalidad del circuito, ya que sin ella los efectos de la anticoagulación sobre la vida del circuito no serán óptimos. En particular nos gustaría remarcar la importancia que tiene colocar el catéter en forma óptima ${ }^{7-9}$ (los autores tienen datos al respecto no publicados).

Una vez establecidos los protocolos y la prescripción que aseguran al mantenimiento de un catéter y un circuito de forma adecuada, se debe considerar agregar un régimen de anticoagulación a la TRRC (opciones de anticoagulación en TRRC están enumerados en la tabla 2). Esto es una consideración a puntualizar ya que, a pesarde que muchos pacientes con LRA presentan un alto riesgo de sangrado, también existe un riesgo importante de complicaciones trombóticas ${ }^{11-13}$. Recordemos que los circuitos extracorpóreos se coagulan con más frecuencia en estos pacientes que en aquellos que tienen una función renal normal11-15. Esto se debe a distintos mecanismos. En primer lugar, la LRA es una entidad caracterizada por una inflamación sistémica con aumento de la

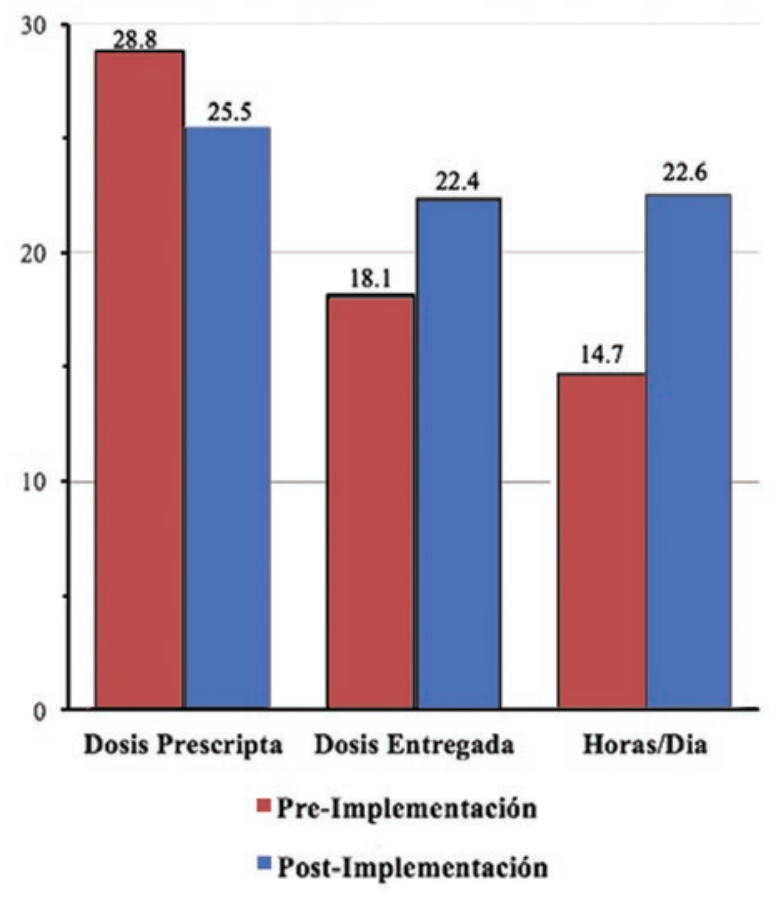

Figura 1. Este gráfico demuestra la diferencia entre la dosis prescripta y la suministrada antes y después de la implementación de un programa integral para la valoración de la calidad de diálisis, con el objeto de mejorar la supervivencia de los circuitos. Los puntos fundamentales que se observan son que la dosis suministrada es de menos del $70 \%$ de la prescripta y esto se debe principalmente a la disminución del tiempo de tratamiento. La implementación del protocolo mejoró todos los parámetros y el mejorar el tiempo de tratamiento nos permitió disminuir la dosis prescripta. Un dato que no se ve en este tipo de análisis es que la supervivencia promedia de los filtros aumentó a más del doble $(46.2 \pm 8.4 \mathrm{~h})$.

producción y disminución de depuración de numerosas factores urémicos y mediadores de inflamación, esta también se acompaña de una pérdida en el equilibrio del equilibrio entre factores procoagulantes y anticoagulantes, y de un aumento de la activación endotelial, que resultan en un medio interno que favorece la coagulación.

Por otra parte, es ampliamente conocido que el circuito extracorpóreo produce un fuerte estímulo procoagulante. El contacto de la sangre con los materiales externos como cánulas, catéteres, tubos, cámaras, agujas y membranas de diálisis activan las vías celulares y moleculares de la cascada de coagulación y resultan en activación de leucocitos y plaquetas ${ }^{16-18}$. Este efecto comienza con el catéter; la colocación y el movimiento del mismo durante el tratamiento pueden causar lesión en la pared del vaso, resultando en activación endotelial y leucocitaria, que, junto al flujo sanguíneo turbulento y altos niveles de presión en el catéter, favorecen una vez más la coagulación ${ }^{19}$. Hay que recalcar que la presencia de recirculación en el 
Tabla 1. Factores primordiales que contribuyen al deterioro prematuro del circuito/membrana en TRRC

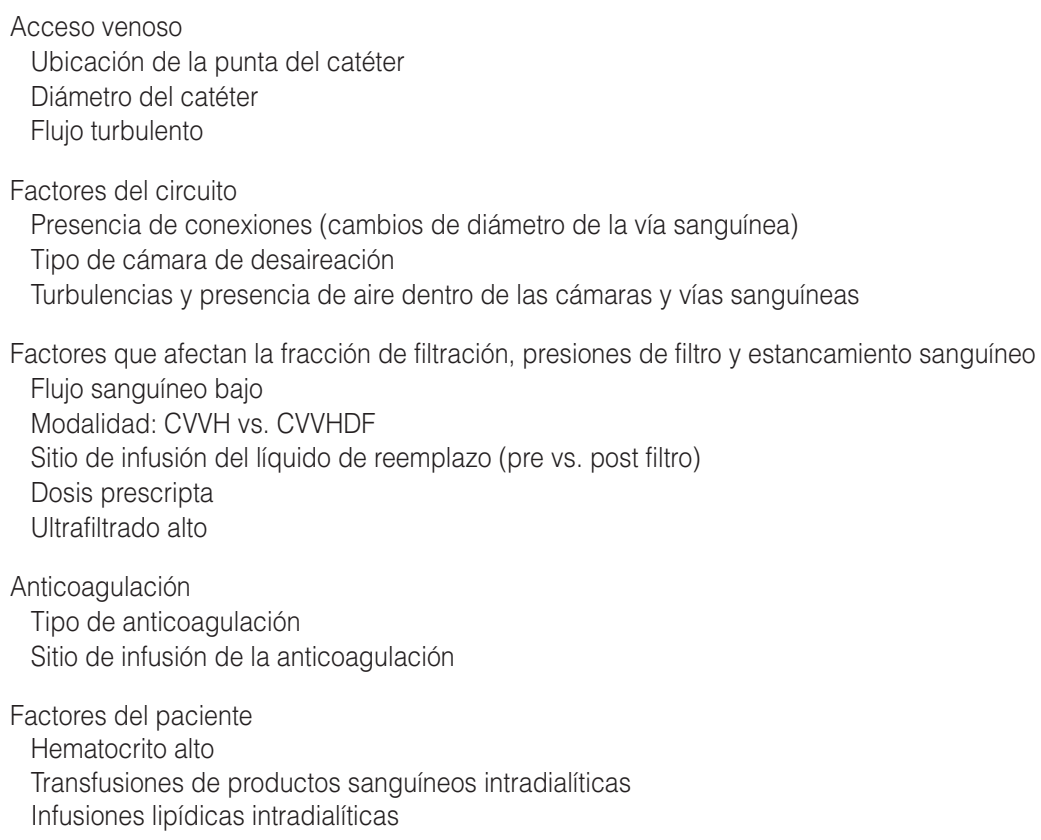

Tabla 2. Opciones de anticoagulación en TRRC

Sin anticoagulación
Heparina no fraccionada
Sistémica
Regional
Heparinas de bajo peso molecular
Citrato para anticoagulación regional
Anticoagulantes alternativos
Prostaglandinas - PGI ${ }_{2}$ PGE
Heparinoides
Dermatán
Danaparoid
Fondaparinux (bolos intermitentes)
Idraparinux (?)
Antagonistas directos de la trombina
Hirudina (no recomendada)
Argatrobán
Inhibidor de serinaproteasa
Maleato de nafamostat (Asia)

acceso puede amplificar esta predisposición trombótica y esto es aplicable por lo menos a todas las terapias dialíticas.

Los procesos procoagulantes que se están estableciendo en el circuito extracorpóreo resultan en una superficie rugosa y pegajosa, rica en micropartículas lipídicas que precipitan la generación de trombina y los depósitos de fibrinas, resultando en un círculo vicioso que termina con la coagulación del circuito. Habrá que tener en cuenta que también existen otros factores que favorecen la formación de trombos durante la terapia de reemplazo renal que podemos controlar (Tabla 1) ${ }^{7-9,20,21}$. En general, cualquier factor que cause un cambio de diámetro dentro de la vía sanguínea, creando zonas de alto flujo y turbulencia, estancamiento de flujo o aquellos que aumentan la interface entre sangre y aire ejercerán un efecto negativo en la supervivencia del circuito. Algunos otros factores que pueden aumentar la viscosidad de la sangre y así predisponer a la coagulación son las fracciones de filtración altas (por flujo sanguíneo bajo o ultrafiltración alto), el hematocrito alto, las transfusiones de productos sanguíneas (paquetes globulares, plaquetas, plas$\mathrm{ma}$, etc.) y las infusiones lipídicas intradialíticas 1,7-9,20.

En conclusión, como todos estos factores suelen encontrarse comúnmente en los pacientes críticos, es necesario tener una estrategia de anticoagulación durante la TRRC para mejorar sus beneficios. El tener una anticoagulación eficaz nos permite proveer una prescripción y dosis adecuada de TRRC. Por último, debemos recordar que esta se ha utilizado recientemente como uno de los estándares de calidad de la prescripción de TRRC, por lo que debe ser un punto de suma atención en el momento de iniciar una terapia continua.

\section{Terapia de reemplazo renal continua sin anticoagulación}

La mayoría de los centros prescriben TRRC sin anticoagulación, particularmente en pacientes con 
alto riesgo de sangrado, o en pacientes con coagulopatías como trombocitopenia o insuficiencia hepática. A pesar de las muchas afirmaciones de su eficacia, hay poca evidencia objetiva de que esta estrategia sea equivalente a los métodos que usan anticoagulación. Si se decide prescribir sin anticoagulación, hay varias pautas que debemos seguir. En primer lugar, se debe asegurar que el cebado inicial del circuito es efectivo y elimina todas las microgotas de aire del circuito y filtro. El cebado puede realizarse con solución salina al $0.9 \%$, sin embargo, es de mayor popularidad usar solución salina heparinizada $(1,000$ 40,000 UI por volumen de cebado; 1-2 litros) para favorecer un acoplamiento de heparina al filtro ${ }^{22,23}$. A pesar del optimismo sobre este método, los datos que demuestran su eficacia son escasos y existen algunos estudios que no encuentran beneficio alguno de esta estrategia en la ausencia de otra forma de anticoagulación. Otra solución de cebado que se ha probado es la albúmina humana ${ }^{24}$. Pero no hay evidencia de eficacia y su costo lo hace prohibitivo para la mayoría de los centros médicos. Estrategias adicionales que pueden mejorar la supervivencia del circuito de TRRC incluyen usar catéteres más anchos, filtros de superficie grandes (aunque esta es muy discutida) y reponer el líquido de reemplazo en forma pre filtro ${ }^{7,20,25-32}$. Este último en particular ha sido reportado con buenos resultados, tanto en su habilidad para aumentar la supervivencia de los circuitos como en disminuir los requerimientos de anticoagulación. Comúnmente se acompaña los métodos mencionados con la administración intermitente de bolos de solución salina al 0.9\%, 50-200 ml cada 30-60 minutos, a pesar de que los resultados obtenidos con esta técnica no han demostrado un beneficio en forma definitiva ${ }^{33-35}$. Finalmente, la composición del filtro quizás influye en la predisposición a la coagulación. Algunos materiales tienen la capacidad de activar a la sangre en forma distinta, disminuyendo esta complicación. No obstante, la significancia clínica de estas diferencias aún no se conoce ${ }^{36}$. Avances en el desarrollo de catéteres, filtros y circuitos que tienen superficies recubiertas por sustancias anticoagulantes, en particular heparina, están siendo examinados en distintas tecnologías extracorpóreas. Actualmente se han probado filtros con membranas recubiertas con heparina o vitamina $E$ que prometen resultados mejores que aquellas terapias prescrita sin anticoagulación ${ }^{20,24}$. Lamentablemente, aún no se tiene la certeza de si estos serán comparables a otras estrategias para prevenir la coagulación.
A pesar de los avances tecnológicos en los biomateriales de las membranas, filtros, líneas y catéteres utilizados para las terapias dialíticas, aún necesitamos del apoyo de los anticoagulantes para minimizar esta reacción con el objetivo de obtener una terapia adecuada, especialmente en los pacientes críticos en los que se encuentra activada la vía de coagulación y existen bajos niveles de anticoagulantes naturales.

\section{Estrategias de anticoagulación para terapia de reemplazo renal continua}

\section{Heparina no fraccionada}

La heparina no fraccionada es un glucosaminoglucano con un peso molecular que varía entre 5 y $100 \mathrm{kDa}$, la mayoría siendo de peso molecular de aproximadamente $30 \mathrm{kDa}$. Tiene una vida media de 30 a 90 minutos, la cual puede aumentar hasta 3 horas en los casos de insuficiencia renal (a pesar de ser preferencialmente metabolizada en forma extrarrenal). La prolongación de su efecto anticoagulante en la insuficiencia renal depende de la retención de los fragmentos más pequeños $(\sim 3 \mathrm{kDa})$, que inhiben la actividad del factor Xa sin afectar al tiempo de tromboplastina parcial (TTP); esta es la razón por la que el aumento de riesgo de sangrado en la insuficiencia renal no se puede predecir siguiendo el TTP. Su mecanismo de acción se basa en su capacidad de unión al ATIII y la inactivación de proteasas séricas (IIa, IXa, y Xa) que resultan en la inhibición de la formación de trombina potenciando la antitrombina y el factor inhibidor XIla.

Por otro lado, el uso de heparina en los pacientes críticos puede tener algunas complicaciones. Obviando la anticoagulación del circuito, también ocasiona anticoagulación sistémica, la cual puede aumentar el riesgo de sangrado, no sin olvidar que las enfermedades críticas pueden predisponer a resistencia a la heparina por bajos niveles de antitrombina, y por otro lado agregamos que la heparina se adhiere a las proteínas de fase aguda, a las células necróticas y apoptósicas, propiciando efectos proinflamatorios que puede disparar la liberación granular por leucocitos polimorfonucleares y plaquetas $^{16}$. Curiosamente, a pesar de su efectividad inconsistente y los riesgos mencionados, sigue siendo el anticoagulante más usado en TRRC $21,24,37$.

Se han desarrollado numerosos protocolos de heparina para la TRRC. La mayoría empiezan con bolos iniciales (p. ej., 10-30 Ul/kg), seguido por infusiones 
continuas (p. ej., 3-20 U/kg/h). La dosis se puede monitorizar y ajustar según el TTP (apuntando a valores entre 1.5-2.5 veces de los normales) o el tiempo de coagulación activada (140-180 segundos) ${ }^{24}$. Algunos han propuesto ajustar para niveles más bajos de TTP; entre 35-45 segundos (1.5 a 2 veces de lo normal), con lo que la vida del filtro podría mantenerse entre 20-40 horas y con menor riesgo de sangrado ${ }^{38}$. El bolo inicial de heparina es frecuentemente reducido a la mitad u omitido completamente en pacientes con alto riesgo de sangrado, aunque la eficacia de este régimen en prevenir sangrados no se ha podido demostrar ${ }^{26}$.

Idealmente, la infusión de heparina se debe administrar lo más cerca posible del acceso vascular y en forma diluida para disminuir su adsorción a las superficies plásticas y maximizar su mezcla con la sangre. A día de hoy, las máquinas de TRRC más frecuentemente utilizadas cuentan con una jeringa que infunde heparina en forma relativamente concentrada a un sitio entre la bomba de sangre y el filtro, dejando todo el circuito extracorpóreo proximal a la bomba de sangre sin anticoagulación.

Una modificación a los protocolos comunes de heparina es aprovechar su reversibilidad con protamina (cada $100 \mathrm{mg}$ de heparina se revierten con $1 \mathrm{mg}$ de protamina). En esto caso se infunde la protamina en el circuito TRRC en un sitio post filtro. El complejo de heparina-protamina circula en forma sistémica, donde el sistema reticuloendotelial lo junta, desacopla y devuelve a la circulación en forma libre. Por lo tanto, es importante monitorear en sitios pre y post infusión de heparina, y post protamina para ajustar el régimen en forma apropiada. Las complicaciones del uso de protamina son la hipotensión, la anafilaxis, la trombocitopenia y, en algunas ocasiones, el sangrado.

\section{Heparina de bajo peso molecular}

Las heparinas de bajo peso molecular (LMWH) están compuestas por los fragmentos más pequeños de la heparina, con pesos moleculares de entre 4.5 y 6 kDA. No tienen actividad sobre la antitrombina (por lo que no prolongan el TTP), pero retienen su actividad sobre el factor $\mathrm{Xa}$; por lo que habrá que monitorizar los niveles de Xa y mantenerlos entre 0.25 y $0.35 \mathrm{U} /$ $\mathrm{ml}$. Cada LMWH tiene características distintas, incluyendo vidas medias que varían comúnmente entre 2 a 4 horas, pero que se pueden prolongar en forma significativa durante la insuficiencia renal, ya que la mayoría son depuradas en forma importante por el riñón. Por lo tanto, requieren ajustes de dosis al caer la función renal ${ }^{20,39,40}$. La más comúnmente utilizada es la enoxaparina, con una dosis de carga de $0.15 \mathrm{mg} /$ $\mathrm{kg}$ y una dosis de mantenimiento $0.05 \mathrm{mg} / \mathrm{kg} / \mathrm{h}$, que puede tener hasta una vida media de $31 \mathrm{~h}$ en el filtro ${ }^{41,42}$. Protocolos usando infusiones o dosis intermitentes de LMWH también se han descrito con tinzaparina, nadroparina y dalteparina ${ }^{40,43-45}$. Dentro de las ventajas que presenta es una anticoagulación efectiva, una farmacocinética predecible, una baja incidencia de trombocitopenia inducida por heparina y un menor efecto sobre los lípidos. Las desventajas son el riesgo de sangrado sistémico (el efecto es solo parcialmente reversible con protamina), que muchos centros no tienen el método para medir el factor anti-Xa disponible y, por último, que los costos son mayores que los de la heparina no fraccionada ${ }^{41,44}$. En caso de sangrado severo por acumulación o sobredosis, se debe administrar plasma y quizás hasta factor VII activado ${ }^{41}$.

\section{Citrato}

El citrato es una pequeña molécula de carga negativa con un peso molecular de $191 \mathrm{Da}$. Su efecto sobre la coagulación se debe a su capacidad de quelar el calcio originando una caída aguda del calcio ionizado (Cai), un cofactor esencial en las tres vías de coagulación, resultando en la inhibición de la formación de trombina. La anticoagulación comienza cuando el Cai cae por debajo de $0.5 \mathrm{mmol} / \mathrm{l}$ y alcanza su eficacia máxima por debajo de $0.25 \mathrm{mmol} / \mathrm{l}^{21,46}$. Además de ser muy efectivo para el almacenamiento de productos sanguíneos, tiene varias características que indican que sería particularmente beneficioso como anticoagulante en sistemas extracorpóreos como plasmaféresis, hemodiálisis y TRRC 21,47,48. En primer lugar, es esencialmente un anticoagulante regional extracorpóreo, por lo cual provee una anticoagulación segura al no aumentar el riesgo de sangra$\mathrm{do}^{4,6}$. Esto se debe a que su efecto anticoagulante se revierte inmediatamente al entrar en la circulación sistémica (por la abundancia de Cai) y a que se metaboliza rápidamente por el hígado, el músculo esquelético y la corteza renal, resultando en una vida media sistémica extremadamente corta ( $\sim 5$ minutos). De la misma manera, la deficiencia de Cai n el circuito inhibe la activación de los granulocitos y plaquetas al contacto con la membrana (aumentando su biocompatibilidad) y disminuye la reacción inflamatoria de los leucocitos y monocitos durante su paso a través del 
Gaceta Médica de México. 2018;Supp 1

Tabla 3. Soluciones comunes de citrato disponibles en forma comercial

\begin{tabular}{lcccc}
\hline Componentes & \multicolumn{4}{c}{ Solución } \\
\cline { 2 - 5 } & 4\% Trisodio citrato & ACD-A 2.2\% Citrato de Na & Prismocitrato 10/2 (Europa) & Prismocitrato 18/0 (Europa) \\
\hline $\mathrm{Na}(\mathrm{mEq} / \mathrm{l})$ & 408 & 224 & 136 & 140 \\
Citrato- $\mathrm{Na}(\mathrm{mmol} / \mathrm{l})$ & 136 & 113 & 10 & 18 \\
Ácido cítrico $(\mathrm{g} / \mathrm{l})$ & & 7.3 & 4.2 & 5,000 \\
Dextrosa $(\mathrm{g} / \mathrm{l})$ & 24.5 & 5,000 & \\
Tamaño de bolsa $(\mathrm{ml})$ & 250 y 500 & 500 y 1000 & &
\end{tabular}

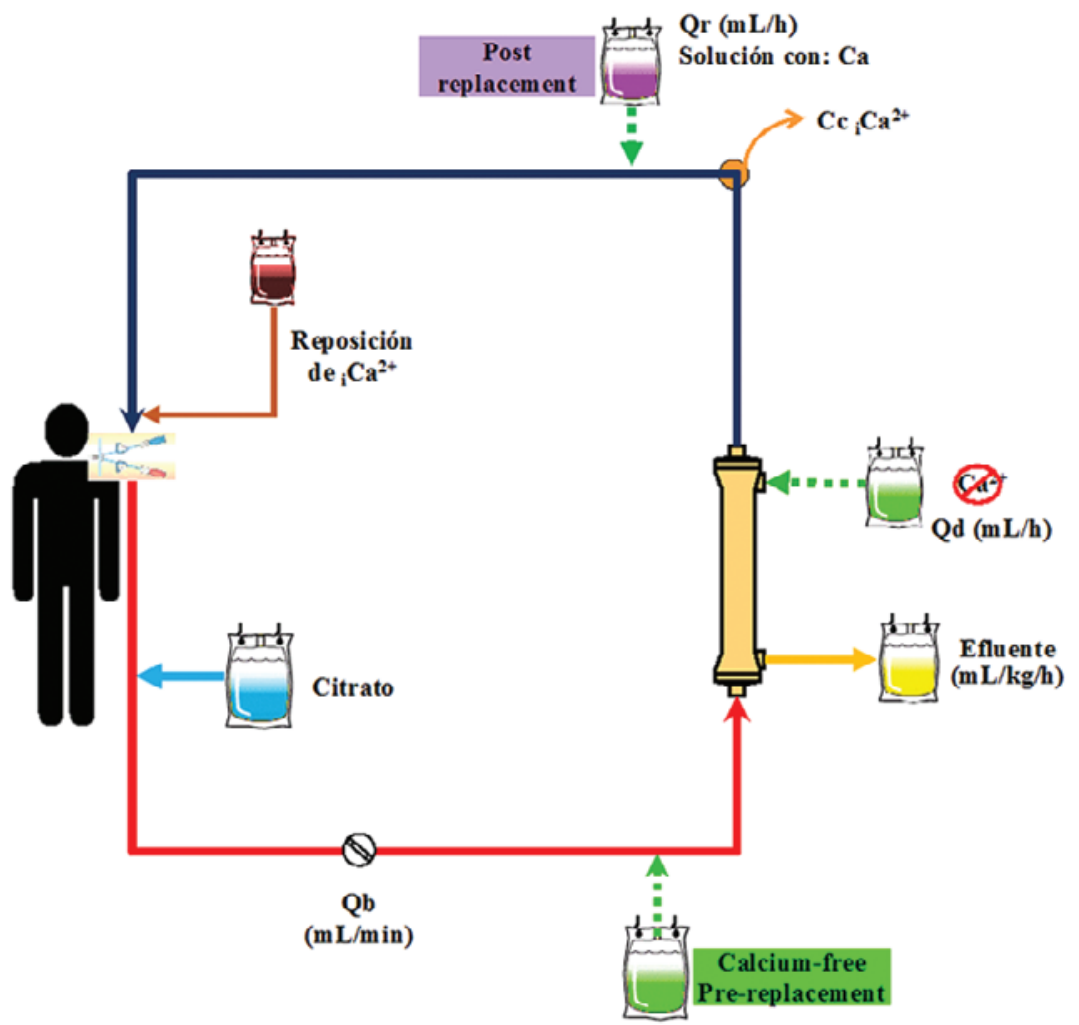

Figura 2. En este diagrama se puede observar como: 1) la aplicación de citrato regional se administra inmediatamente al inicio del circuito previo a la bomba de sangre, durante este trayecto la solución dializante que circulará en el circuito y a través del filtro estará libre de Ca (Pre-filtro); 2) los niveles de Cai deben medirse en el post filtro en algunos casos, principalmente para realizar una adecuada reposición en los pacientes pediátricos, esto debe hacerse de manera cercana a la reinfusión del paciente; 3) la solución de reemplazo post filtro debe utilizar calcio; y 4) por último, no debemos olvidar la pérdida importante de Ca en el efluente que también requiere reposición.

Qb: flujo de bomba de sangre; Qd: flujo de bomba de diálisis; Qr: flujo de bomba de reemplazo; Cc iCa2+: Calcio ionizado en el circuito (post filtro); CVVHDF: hemodiafiltración venovenosa continua.

circuito extracorpóreo $21,49-51$. O sea, la anticoagulación regional con citrato $(R C A)$ sería potencialmente de mayor beneficio en estos pacientes críticos tratados con TRRC. Lamentablemente, el uso de citrato en la TRRC ha crecido muy lentamente a pesar de los adelantos en los protocolos.

Hay varias fórmulas de soluciones de citrato que se pueden usar ${ }^{52}$. En los Estados Unidos, las fórmulas utilizadas son la solución de $4 \%$ citrato trisódico o
ACDA (solución anticoagulante de dextrosa-citrato), mientras en Europa disponen de soluciones de citrato más fisiológicas (Prismocitrate®) (Tabla 3). El citrato se infunde en la parte inicial del circuito extracorpóreo (Fig. 2) en proporción al flujo sanguíneo para que los niveles de citrato alcancen entre 4 a $6 \mathrm{mmol} / \mathrm{l}$, que es suficiente para reducir el nivel de Cai en el circuito por debajo de $0.35 \mathrm{mmo}^{21,22}$. Además, algunos aconsejan medir el Cai post filtro y ajustar la velocidad de 
flujo de citrato hasta alcanzar los niveles deseados. Una vez que estos niveles son alcanzados, los flujos sanguíneos y de citrato deben cambiarse en conjunto en forma proporcional para mantener los niveles óptimos de citrato/Cai.

El complejo formado por el citrato-calcio transitará hacia uno de dos destinos, se puede filtrar y perder por el efluente (es muy pequeño, por lo cual se filtra libremente) o entrar en la circulación sistémica, donde es metabolizado por los tejidos. La fracción metabolizada liberará Cai (ayudando a reponer los niveles de Cai sérico) y se formarán tres moléculas de bicarbonato por cada molécula de citrato, siendo esta entonces una fuente importante de bicarbonato. En cambio, la fracción de calcio perdido en el efluente debe reponerse para evitar que el paciente desarrolle hipocalcemia. La reposición puede realizarse con cloruro de calcio o gluconato de calcio. El segundo tiene la ventaja de que puede infundirse a través de una vía periférica, aunque hay que recordar que esta será una infusión continua y puede ser altamente tóxico si la vía se infiltra ${ }^{53,54}$. En nuestra experiencia, la reposición de calcio se realiza por la vía del retorno venoso del circuito para evitar someter al paciente a otra vía central (u ocupar en forma innecesario una vía). A pesar de la posibilidad de que se restituya la coagulación mientras la sangre sigue en el circuito extracorpóreo, no hemos detectado ningún aumento en la trombosis del acceso ni cambios en nuestra supervivencia de circuitos.

Los protocolos de RCA en que ajustan las infusiones en base a los niveles de Cai pos-filtro suelen resultar en ajustes frecuentes de las infusiones de citrato y el calcio. Esto tiene varias consecuencias importantes, además de someter al paciente a flebotomías frecuentes, aumenta el trabajo/frustración de las enfermeras y médicos, los costos, y el potencial de cometer errores. Muchos médicos han concluido que no es necesario porque al estandarizar los protocolos, uno encuentra que los valores de Cai pos-filtro varían muy poco. Además, el resultado que más relevancia tiene es si el filtro se coagula, no el nivel de Cai pos-filtro. Por lo tanto, muchos usamos protocolos simplificados de RCA en los que la dosis de citrato es relativamente fija y no medimos el Cai en el circuito en forma sistemática ${ }^{37,55}$; solo lo hacemos si tenemos coagulación del circuito prematura. Hasta ahora hemos detectado niveles de Cai alto en menos del $3 \%$ de los casos.

Hay dos formas en las que un centro puede implementar protocolos de RCA. En el primero, la RCA se reserva solo para aquellos pacientes con riesgo elevado de sangrado como cirugía o trauma reciente, diátesis hemorrágica, lesiones intracraneales, pericarditis o retinopatía urémica, hipertensión maligna, etc. La segunda forma es la de estandarizar el programa para que se use en todos aquellos que lo toleran. Se prefiere esta última por las siguientes razones: primero, hay por lo menos siete estudios aleatorizados y controlados que compararon RCA con heparina o heparina de bajo peso molecular y la mayoría demostraron superioridad en eficacia o seguridad con RCA ${ }^{56-61}$; segundo, al usar el mismo protocolo para casi todos los pacientes se minimizan las posibilidades de errores importantes y aumenta la eficiencia; tercero, el uso de RCA ha sido recomendado por el grupo Kidney Disease Improving Global Outcomes (KDIGO 2012), la Canadian Society of Nephrology y la Italian Society of Nephrology. En particular, hay que notar que el grupo KDIGO sugiere que se use RCA en vez de heparina en pacientes sin riesgo de sangrado (el nivel de evidencia fue de $2 \mathrm{~B}$ ) y RCA en vez de TRRC sin anticoagulación en pacientes con riesgo de sangrado $(2 \mathrm{C})^{62}$.

Una razón por el cual el uso de RCA no se ha extendido más es el temor a las complicaciones metabólicas, siendo las más frecuentes hipocalcemia, hipernatremia, hipomagnesemia, acidosis y alcalosis metabólica. Si bien hay que mantenerse a la expectativa de estos, la implementación de protocolos validados y de sistemas de monitoreo ha minimizado la incidencia de estas complicaciones. La hipocalcemia es generalmente causada por un reemplazo deficiente o la suspensión inapropiada de la infusión de calcio. La hipernatremia y la alcalosis metabólica usualmente se deben a infusiones excesivas de la solución de citrato o de dosis insuficiente de TRRC, otra opción es la deficiente infusión de soluciones hipotónicas (si la solución de citrato es al $4 \%$ de trisodio citrato). El citrato también quela magnesio ocasionalmente, causando hipomagnesemia ${ }^{63}$. El citrato puede causar acidosis mediante su contribución a los iones fuertes (similar a salina), pero también puede acumularse en condiciones en que su metabolismo está comprometido, particularmente la insuficiencia hepática fulminante. Esto causa acumulación de citrato que se caracteriza por el llamado citrate lock. Las características bioquímicas que nos pueden ayudar a identificar esta condición incluyen la disminución del Cai sérico sistémico, la disminución del pH arterial con brecha aniónica elevada, el calcio sérico total elevado y una brecha de calcio alta (Ca sérico 


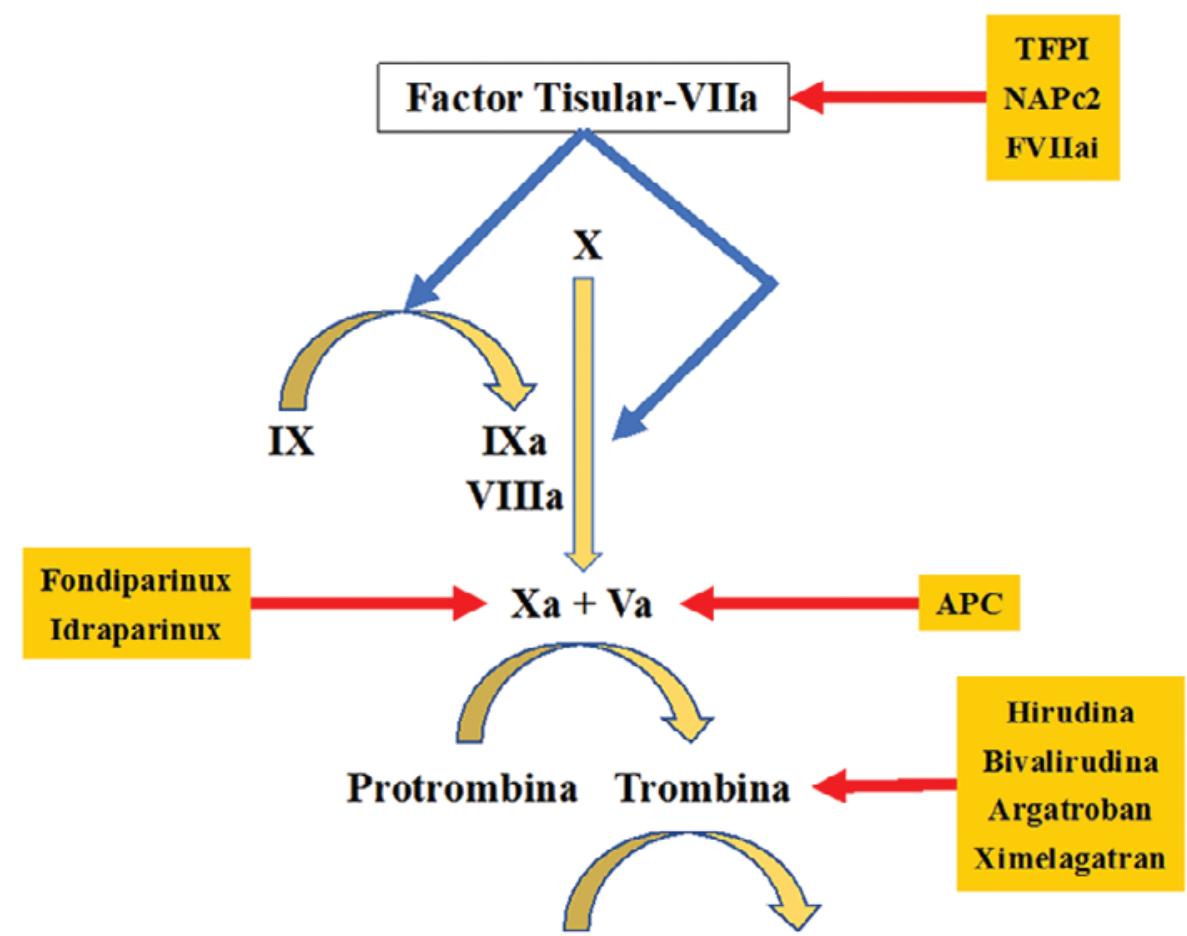

Fibrinógeno Fibrina

Figura 3. En algunos casos, el uso de heparina no fraccionada o citrato no es posible. En estos casos se puede hacer uso de otras alternativas de anticoagulación, algunas de las cuales se muestran en la figura 2 actuando a distintos niveles de la cascada de coagulación: 1) el inhibidor de la vía del factor tisular (TFPI), la proteína C2 del nematodo anticoagulante (NAPC2) y el inhibidor del factor 7 activado actúan sobre el factor tisular VIla; 2) los heparinoides como el fondiparinux y el idraparinux principalmente inhiben al factor Xa no permitiendo el paso de protrombina a trombina; 3) los inhibidores de la trombina como la hirudina, la bivalirudina, el argatrobán y el ximelgatrán no permiten el paso del fibrinógeno a fibrina, evitando así la formación del coágulo.

total menos Cai) o un índice calcio total (mg/dl) entre un Cai (mmol/l) mayor de $10^{21,37,52}$. El tratamiento principal se basa en evitar o corregir la hipocalcemia (ionizada), ya que esta anormalidad produce los trastornos más significativos. La presencia de una brecha de calcio en ausencia de niveles bajos de Cai es de significancia desconocida. No obstante, si la brecha de calcio es severa, puede ser beneficioso disminuir la infusión de citrato o aumentar la dosis de TRRC para acelerar la depuración de citrato (después de reponer el Cai).

Las condiciones clínicas que pueden predisponer a la toxicidad por citrato son: insuficiencia hepática fulminante 0 , más infrecuentemente, cirrosis crónica avanzada, choque cardiogénico (por hipoperfusión hepática y del músculo estriado), uso de anfetaminas, pancreatitis grave, síndrome de lisis tumoral, el envenenamiento por etilenglicol e inhibidores de la cadena respiratoria (cianuro), citopatías mitocondriales y el uso de antiretrovirales ${ }^{21}$. En estas condiciones se debe usar citrato con más cuidado, quizás aumentando la dosis de TRRC (para aumentar su depuración por esta vía), y monitoreando los análisis de laboratorio más frecuentemente. Una última característica que tener en cuenta en relación con el citrato, aunque no es una complicación, es su capacidad de conferir energía, $3 \mathrm{kcal} / \mathrm{g}(0.59 \mathrm{kcal} / \mathrm{mmol})$. Un día de uso de citrato en TRRC puede aportar entre 350-500 Kcal dependiendo de la modalidad.

El uso de citrato como anticoagulante tiene beneficios potenciales significativos para pacientes críticos en TRRC. Su eficacia y seguridad están comprobados; prolonga la vida media de los circuitos de TRRC y resulta en menos episodios de sangrados importante y en el número de unidades de sangre transfundidas. La introducción de protocolos simples ha minimizado el riesgo de complicaciones, lo cual permite su uso en forma más generalizada. Por estas razones está ganando popularidad y estableciéndose como el método preferido de anticoagulación para TRRC (la tabla 4 muestra la comparación entre ventajas y desventajas del RCA y la heparina no fraccionada). 
Tabla 4. Las ventajas y desventajas del uso de heparina y el citrato como anticoagulante

\begin{tabular}{ll}
\hline Heparina no fraccionada & Desventajas \\
\hline Ventajas & Sangrado sistémico \\
\hline Fácil acceso & Cinética impredecible \\
Económica & Resistencia a la heparina por bajos niveles de antitrombina \\
Efectiva & Trombocitopenia inducida por la heparina \\
Monitorización sencilla con TTP & Niveles de aPTT no predicen sangrados \\
Vida media corta & \\
\hline Fácil reversibilidad con protamina & \\
\hline Citrato & Desventajas \\
\hline Ventajas & Riesgo de hipocalcemia sistémica \\
\hline Menos riesgo de sangrado & Riesgo de hipernatremia \\
Mejor tolerancia & Riesgo de alcalosis metabólica \\
Prolonga la vida media del circuito & Protocolos suelen ser mas complejo \\
Mejora la dosis de TRRC & Monitoreo puede ser mas cara en algunos protocolos \\
\hline Mejora la biocompatibilidad &
\end{tabular}

aPTT: tiempo de tromboplastina parcial activado; TTP: tiempo de tromboplastina parcial; TRRC: terapia de reemplazo renal continuo.

\section{Otras opciones de anticoagulación}

Existen circunstancias particulares en las cuales el uso de heparina o citrato no es deseable o está francamente contraindicado y sin embargo la necesidad de la anticoagulación para mantener la supervivencia del filtro es indispensable. Existen una variedad de opciones de anticoagulantes que se pueden utilizar en estas condiciones (Tabla 2 y Fig. 3). Algunas de estas opciones son remotamente utilizadas, como las prostaglandinas $\mathrm{PGI}_{2}$ y $\mathrm{PGE}_{1}$, que aprovechan de la función de inhibición de la adhesión y agregación plaquetaria, tienen una vida media de dos minutos y su efecto antiplaquetario es de alrededor de dos horas ${ }^{64}$. Su protocolo de uso es una infusión pre filtro de $2-8 \mathrm{ng} / \mathrm{kg} / \mathrm{min}$. Los prostanoides no afectan la cascada de coagulación (por lo tanto, no se puede monitorear su eficacia con las pruebas comunes de laboratorio) y sus efectos antitrombóticos son mayormente limitados al circuito. Los mejores resultados se obtuvieron cuando se combinaron con dosis bajas de heparina. Entre sus desventajas está el riesgo de hipotensión (por su efecto vasodilatador) y el alto costo ${ }^{64-67}$.

Otras opciones incluyen los heparinoides como el dermatán $^{68}$, el danaparoid ${ }^{69,70}$, el fondiparinux y el idraparinux, que actúan principalmente sobre el factor anti-Xa pero ejerciendo un efecto menor sobre el Ila, y los inhibidores directos de trombina como la hirudina, bivalirudina, argatrobán ${ }^{71}$ y ximelgatrán, que no permiten la conversión del fibrinógeno a fibrina, evitando así la formación del coágulo. Se han descrito diversos protocolos que se pueden utilizar con estas sustancias y estudios pequeños han demostrado buena eficacia, generalmente sin un aumento mayor en el riesgo de sangrado. A pesar de esto, tienen un rol incierto en la TRRC, ya que su costo es prohibitivo y no presentan grandes ventajas sobre los anticoagulantes más tradicionales excepto en contados pacientes. Además, algunos tienen características problemáticas que dificulta su uso en TRRC. Por ejemplo, los heparinoides sintéticos (fondiparinux e idraparinux) tienen vidas medias muy prolongadas, lo que impide que se usen en forma de infusión continua por el riesgo de acumulación de fármaco que aumentaría el riesgo de sangrado. En forma similar, la vida media de la hirudina aumenta desede 1-2 horas hasta 50 horas en pacientes anéfricos. Además, el uso prolongado de este fármaco puede estimular la creación de anticuerpos antihirudina que no solo prolongan aún más su vida media, sino también a su actividad. Finalmente, hay que tener en cuenta que no existe antídoto para revertir los efectos en la mayoría de estos factores, aunque se han reportado algunos éxitos con factor VII activado $^{72-74}$. 


\section{Conclusión}

La coagulación prematura del circuito extracorpóreo de la TRRC sigue siendo una barrera al uso óptimo de esta modalidad de reemplazo renal. Este fallo en la provisión de TRRC no solo causa pérdidas evitables de sangre, sino también un suministro inadecuado del tratamiento y aumento de los costos, consumo de recursos, incremento en el trabajo y frustraciones del personal de salud que cuida del paciente. Por lo que es necesario mejorar la supervivencia de los circuitos e implementar un plan integral que incorpore medidas que optimicen los factores del circuito (acceso vascular), la prescripción y la anticoagulación. Actualmente el anticoagulante más utilizado en la TRRC es la heparina, sin embargo, se ha visto una superioridad en la vida media del filtro y disminución de riesgo de sangrado con la anticoagulación regional con citrato. Los protocolos modernos son muy simples, efectivos y de bajos costos. Además, son tolerados por casi todos los pacientes, incluyendo a la mayoría de aquellos con insuficiencia hepática. Sin embargo, existen situaciones en que el médico necesita recurrir a otra estrategia que dependerá de las características individuales del paciente y los recursos disponibles, por lo que los tratamientos son individuales y dinámicos.

\section{Bibliografía}

1. Connor MJ Jr, Karakala N. Continuous renal replacement therapy: reviewing current best practice to provide high-quality extracorporeal therapy to critically ill patients. Adv Chronic Kidney Dis. 2017;24;213-8.

2. Golper TA. Hybrid renal replacement therapies for critically ill patients. Contrib Nephrol. 2004;144;278-83.

3. Manns B, Doig CJ, Lee H, Dean S, Tonelli M, Johnson D, et al. Cost of acute renal failure requiring dialysis in the intensive care unit: clinical and resource implications of renal recovery. Crit Care Med. 2003; 31;449-55.

4. Pannu N, Gibney RN. Renal replacement therapy in the intensive care unit. Ther Clin Risk Manag. 2005:1;141-50.

5. Rewa OG, Villeneuve PM, Lachance P, Eurich DT, Stelfox HT, Gibney RTN, et al. Quality indicators of continuous renal replacement therapy (CRRT) care in critically ill patients: a systematic review. Intensive Care Med. 2017;43;750-63

6. Rewa O, Villeneuve PM, Eurich DT, Stelfox HT, Gibney RT, Hartling L, et al. Quality indicators in continuous renal replacement therapy (CRRT) care in critically ill patients: protocol for a systematic review. Syst Rev. 2015;4;102.

7. Baldwin I. Factors affecting circuit patency and filter 'life'. Contrib Nephrol. 2007;156;178-84.

8. Zhang L, Tanaka A, Zhu G, Baldwin I, Eastwood GM, Bellomo R. Patterns and mechanisms of artificial kidney failure during continuous renal replacement therapy. Blood Purif. 2016;41;254-63.

9. Baldwin I, Bellomo R. Relationship between blood flow, access catheter and circuit failure during CRRT: a practical review. Contrib Nephrol. 2004;144;203-13.

10. Venkataraman R, Kellum JA, Palevsky P. Dosing patterns for continuous renal replacement therapy at a large academic medical center in the United States. J Crit Care. 2002;17;246-50.

11. Davenport $A$. The coagulation system in the critically ill patient with acute renal failure and the effect of an extracorporeal circuit. Am J Kidney Dis. 1997;30;:S20-7.

12. Shulman RI, Singer M, Rock J. Continuous renal replacement therapy. Keeping the circuit open: lessons from the lab. Blood Purif. 2002;20;275-81.
13. Al-Dorzi HM, Al-Heijan A, Tamim HM, Al-Ghamdi G, Arabi YM. Renal failure as a risk factor for venous thromboembolism in critically III patients: a cohort study. Thromb Res. 2013;132;671-5.

14. Davenport A. Anticoagulation for acute dialysis. En: Jörres $A$, Ronco $C$ Kellum JA, editores. Management of acute kidney problems. Springer-Verlag Berlin Heidelberg; 2010. pp. 559-75.

15. Davenport A. What are the anticoagulation options for intermittent hemodialysis? Nat Rev Nephrol. 2011;7;499.

16. Bellomo R, Ronco C, Mehta RL, Asfar P, Boisrame-Helms J, Darmon M, et al. Acute kidney injury in the ICU: from injury to recovery: reports from the $5^{\text {th }}$ Paris International Conference. Ann Intensive Care. 2017;7;49.

17. Annich GM. Extracorporeal life support: the precarious balance of hemostasis. J Thromb Haemost. 2015;13 Suppl 1;S336-42.

18. Millar JE, Fanning JP, McDonald $\mathrm{Cl}$, McAuley DF, Fraser JF. The inflammatory response to extracorporeal membrane oxygenation (ECMO): a review of the pathophysiology. Crit Care. 2016;20;387.

19. Davenport A. Central venous catheters for hemodialysis: How to overcome the problems. Hemodial Int. 2000;4;78-82.

20. Joannidis M, Oudemans-van Straaten HM. Clinical review: Patency of the circuit in continuous renal replacement therapy. Crit Care. 2007;11;218.

21. Davenport A, Tolwani A. Citrate anticoagulation for continuous renal replacement therapy (CRRT) in patients with acute kidney injury admitted to the intensive care unit. NDT Plus. 2009;2;439-47.

22. Tolwani AJ, Prendergast MB, Speer RR, Stofan BS, Wille KM. A practical citrate anticoagulation continuous venovenous hemodiafiltration protocol for metabolic control and high solute clearance. Clin J Am Soc Nephrol. 2006;1;79-87.

23. Sahota S, Rodby R. Inpatient hemodialysis without anticoagulation in adults. Clin Kidney J. 2014;7;552-6.

24. Nongnuch A, Tangsujaritvijit V, Davenport A. Anticoagulation for renal replacement therapy for patients with acute kidney injury. Minerva Urol Nefrol. 2016;68:87-104.

25. González de Molina FJ, Galindo M, González C, Broch MJ, Del Bano L, Roglan A. Vascular access and extracorporeal circuit patency in continuous renal replacement therapy. Med Intensiva. 2016;40;572-85.

26. Bellomo $\mathrm{R}$, Teede $\mathrm{H}$, Boyce $\mathrm{N}$. Anticoagulant regimens in acute continuous hemodiafiltration: a comparative study. Intensive Care Med. 1993; 19;329-32.

27. Dungen HD, von Heymann C, Ronco C, Kox WJ, Spies CD. Renal replacement therapy: physical properties of hollow fibers influence efficiency. Int J Artif Organs. 2001;24;357-66.

28. Kumar VA, Yeun JY, Depner TA, Don BR. Extended daily dialysis vs. continuous hemodialysis for ICU patients with acute renal failure: a twoyear single center report. Int J Artif Organs. 2004;27;371-9.

29. Honore $\mathrm{P}$, Wittebole $X$, Lozano A. Evaluation of the predilution technique in reducing the occurrence of bleeding during continuous venovenous haemofiltration in critically ill patients. Efficacy of predilution in reducing the amount of anticoagulation during CVVH. Critical Care 1997;1;P071.

30. Kaplan AA. Predilution versus postdilution for continuous arteriovenous hemofiltration. Trans Am Soc Artif Intern Organs. 1985;31;28-32.

31. Uchino S, Fealy N, Baldwin I, Morimatsu H, Bellomo R. Pre-dilution vs. post-dilution during continuous veno-venous hemofiltration: impact on filter life and azotemic control. Nephron Clin Pract. 2003;94;c94-8.

32. van der Voort PH, Gerritsen RT, Kuiper MA, Egbers PH, Kingma WP, Boerma EC. Filter run time in CVVH: pre- versus post-dilution and nadroparin versus regional heparin-protamine anticoagulation. Blood Purif. 2005;23;175-80.

33. Nagarik AP, Soni SS, Adikey GK, Raman A. Comparative study of anticoagulation versus saline flushes in continuous renal replacement therapy. Saudi J Kidney Dis Transpl. 2010;21;478-83.

34. Panphanpho S, Naowapanich $S$, Ratanarat R. Use of saline flush to prevent filter clotting in continuous renal replacement therapy without anticoagulant. J Med Assoc Thai. 2011;94 Suppl 1;S105-10.

35. Ramesh Prasad GV, Palevsky PM, Burr R, Lesko JM, Gupta B, Greenberg $A$. Factors affecting system clotting in continuous renal replacement therapy: results of a randomized, controlled trial. Clin Nephrol. 2000;53; 55-60.

36. Chanard J, Lavaud S, Randoux C, Rieu P. New insights in dialysis membrane biocompatibility: relevance of adsorption properties and heparin binding. Nephrol Dial Transplant. 2002:18:252-7.

37. Oudemans-van Straaten HM, Kellum JA, Bellomo R. Clinical review: anticoagulation for continuous renal replacement therapy--heparin or citrate? Crit Care. 2011;15;202.

38. van de Wetering J, Westendorp RG, van der Hoeven JG, Stolk B, Feuth JD, Chang PC. Heparin use in continuous renal replacement procedures: the struggle between filter coagulation and patient hemorrhage. J Am Soc Nephrol. 1996;7;145-50.

39. Bhattarai M, Rajapakase R, Palevsky PM. Continuous renal replacement therapies (CRRT) Overview. En: Magee CC, Tucker JK, Singh AK. editores. Core concepts in dialysis and continuous therapies. Springer US; 2016. pp. 191-203.

40. de Pont AC, Oudemans-van Straaten HM, Roozendaal KJ, Zandstra DF. Nadroparin versus dalteparin anticoagulation in high-volume, continuous 
venovenous hemofiltration: a double-blind, randomized, crossover study. Crit Care Med. 2000;28;421-5.

41. Joannidis $M$, Kountchev J, Rauchenzauner M, Schusterschitz N Ulmer $\mathrm{H}$, Mayr A, et al. Enoxaparin vs. unfractionated heparin for anticoagulation during continuous veno-venous hemofiltration: a randomized controlled crossover study. Intensive Care Med. 2007;33;1571-9.

42. Wynckel A, Bernieh B, Toupance O, N'Guyen PH, Wong T, Lavaud S et al. Guidelines to the use of enoxaparin in slow continuous hemodialysis. Contrib Nephrol. 1991;93;221-4.

43. Jeffrey RF, Khan AA, Douglas JT, Will EJ, Davison AM. Anticoagulation with low molecular weight heparin (Fragmin) during continuous hemodialysis in the intensive care unit. Artif Organs. 1993;17;717-20.

44. Reeves JH, Cumming AR, Gallagher L, O'Brien JL, Santamaria JD. A controlled trial of low-molecular-weight heparin (dalteparin) versus unfractionated heparin as anticoagulant during continuous venovenous hemodialysis with filtration. Crit Care Med. 1999;27;2224-8.

45. Voiculescu M IG, Ionescu C. Anticoagulation efficacy and safety with a low molecular weight heparin-tinzaparin in continous renal replacecment therapies. Blood Purif. 2002;20.

46. Sakariassen KS, Ottenhof-Rovers M, Sixma JJ. Factor VIII-von Willebrand factor requires calcium for facilitation of platelet adherence. Blood. 1984;63;996-103.

47. Flanigan MJ, Pillsbury L, Sadewasser G, Lim VS. Regional hemodialysis anticoagulation: hypertonic tri-sodium citrate or anticoagulant citrate dextrose-A. Am J Kidney Dis. 1996;27;519-24.

48. Pinnick RV, Wiegmann TB, Diederich DA. Regional citrate anticoagulation for hemodialysis in the patient at high risk for bleeding. $N$ Engl $J$ Med. 1983;308;258-61.

49. Bos JC, Grooteman MP, van Houte AJ, Schoorl M, van Limbeek J, Nubé MJ. Low polymorphonuclear cell degranulation during citrate anticoagulation: a comparison between citrate and heparin dialysis. Nephro Dial Transplant. 1997;12;1387-93.

50. Dhondt A, Vanholder R, Tielemans C, Glorieux G, Waterloos MA, De Smet $R$, et al. Effect of regional citrate anticoagulation on leukopenia, complement activation, and expression of leukocyte surface molecules during hemodialysis with unmodified cellulose membranes. Nephron. 2000;85;334-42.

51. Berridge MJ, Bootman MD, Roderick HL. Calcium signalling: dynamics homeostasis and remodelling. Nat Rev Mol Cell Biol. 2003;4;517-29.

52. Oudemans-van Straaten HM, Ostermann M. Bench-to-bedside review: Citrate for continuous renal replacement therapy, from science to practice. Crit Care. 2012;16;249.

53. Martin TJ, Kang Y, Robertson KM, Viri MA, Marquez JM. Ionization and hemodynamic effects of calcium chloride and calcium gluconate in the absence of hepatic function. Anesthesiology. 1990;73;62-5.

54. Lake C, Beecroft CL. Extravasation injuries and accidental intra-arterial injection. Continuing Education in Anaesthesia Critical Care \& Pain. 2010;10;109-13.

55. Oudemans-van Straaten HM. Citrate anticoagulation for continuous renal replacement therapy in the critically ill. Blood Purif. 2010;29;191-6.

56. Fealy N, Baldwin I, Johnstone M, Egi M, Bellomo R. A pilot randomized controlled crossover study comparing regional heparinization to regional citrate anticoagulation for continuous venovenous hemofiltration. Int $J$ Artif Organs. 2007;30;301-7.

57. Betjes MG, van Oosterom D, van Agteren M, van de Wetering J. Regional citrate versus heparin anticoagulation during venovenous hemofiltration in patients at low risk for bleeding: similar hemofilter survival but significantly less bleeding. J Nephrol. 2007;20;602-8.

58. Stucker F, Ponte B, Tataw J, Martin P-Y, Wozniak H, Pugin J, et al Efficacy and safety of citrate-based anticoagulation compared to heparin in patients with acute kidney injury requiring continuous renal replacement therapy: a randomized controlled trial. Critical Care. 2015; $19 ; 91$.

59. Hetzel GR, Schmitz M, Wissing H, Ries W, Schott G, Heering PJ, et al. Regional citrate versus systemic heparin for anticoagulation in critically ill patients on continuous venovenous haemofiltration: a prospective randomized multicentre trial. Nephrol Dial Transplant. 2011; $26 ; 232-9$.

60. Kutsogiannis DJ, Gibney RT, Stollery D, Gao J. Regional citrate versus systemic heparin anticoagulation for continuous renal replacement in critically ill patients. Kidney Int. 2005;67;2361-7.

61. Monchi M, Berghmans D, Ledoux D, Canivet JL, Dubois B, Damas P. Citrate vs. heparin for anticoagulation in continuous venovenous hemofiltration: a prospective randomized study. Intensive Care Med. 2004;30;260-5.

62. Group KDIGOKAKIW. KDIGO Clinical Practice Guideline for Acute Kidney Injury. Kidney inter, Supp 2012;2;124-138

63. Brain M, Anderson M, Parkes S, Fowler P. Magnesium flux during continuous venovenous haemodiafiltration with heparin and citrate anticoagulation. Crit Care Resusc. 2012;14(4):274-82.

64. Arcangeli A, Rocca B, Salvatori G, Ciancia M, De Cristofaro R, AntoneIli M. Heparin versus prostacyclin in continuous hemodiafiltration for acute renal failure: effects on platelet function in the systemic circulation and across the filter. Thromb Res. 2010;126;24-31.

65. Langenecker SA, Felfernig M, Werba A, Mueller CM, Chiari A, Zimpfer M. Anticoagulation with prostacyclin and heparin during continuous venovenous hemofiltration. Crit Care Med. 1994;22;1774-81.

66. Kozek-Langenecker SA, Kettner SC, Oismueller C, Gonano C, Speiser W, Zimpfer M. Anticoagulation with prostaglandin E1 and unfractionated heparin during continuous venovenous hemofiltration. Crit Care Med. 1998;26;1208-12.

67. Kozek-Langenecker SA, Spiss CK, Gamsjager T, Domenig C, Zimpfer M. Anticoagulation with prostaglandins and unfractionated heparin during continuous venovenous haemofiltration: a randomized controlled trial. Wien Klin Wochenschr. 2002;114;96-101.

68. Ryan KE, Lane DA, Flynn A, Ireland H, Boisclair M, Shepperd J, et al. Antithrombotic properties of dermatan sulphate (MF 701) in haemodialysis for chronic renal failure. Thromb Haemost. 1992;68;563-9.

69. Lindhoff-Last E, Betz C, Bauersachs R. Use of a low-molecular-weight heparinoid (danaparoid sodium) for continuous renal replacement therapy in intensive care patients. Clin Appl Thromb Hemost. 2001;7; 300-4.

70. de Pont AC, Hofstra JJ, Pik DR, Meijers JC, Schultz MJ. Pharmacokinetics and pharmacodynamics of danaparoid during continuous venovenous hemofiltration: a pilot study. Crit Care. 2007;11;R102.

71. Treschan TA, Schaefer MS, Geib J, Bahlmann A, Brezina T, Werner P, et al. Argatroban versus Lepirudin in critically ill patients (ALicia): a randomized controlled trial. Crit Care. 2014;18;588.

72. Bijsterveld NR, Moons AH, Boekholdt SM, van Aken BE, Fennema H, Peters RJ, et al. Ability of recombinant factor VIla to reverse the anticoagulant effect of the pentasaccharide fondaparinux in healthy volunteers. Circulation. 2002;106;2550-4

73. Lisman T, Bijsterveld NR, Adelmeijer J, Meijers JC, Levi M, Nieuwenhuis HK, et al. Recombinant factor VIla reverses the in vitro and ex vivo anticoagulant and profibrinolytic effects of fondaparinux. J Thromb Haemost. 2003;1;2368-73.

74. Young G, Yonekawa KE, Nakagawa PA, Blain RC, Lovejoy AE, Nugent DJ. Recombinant activated factor VII effectively reverses the anticoagulant effects of heparin, enoxaparin, fondaparinux, argatroban, and bivalirudin ex vivo as measured using thromboelastography. Blood Coagul Fibrinolysis. 2007;18;547-53. 